\title{
Rejuvenation of $\beta$ cells by epigenetic editing
}

\author{
Tao Wang ${ }^{1,2,3}$ and Duanqing Pei $\mathrm{i}^{1,2,3,4}$ \\ 'Guangzhou Institutes of Biomedicine and Health, ${ }^{2}$ CAS Key Laboratory of Regenerative Biology, Guangzhou, China. ${ }^{3}$ Guangdong Provincial Key Laboratory of Stem Cell and Regenerative Medicine, \\ ${ }^{4}$ Guangzhou Regenerative Medicine and Health, Guangdong Laboratory, Guangzhou, China.
}

\begin{abstract}
Insulin-secreting $\beta$ cell loss or dysfunction is a feature of both type 1 and type 2 diabetes. Strategies to restore $\beta$ cell mass are limited, as sources of healthy islets are scarce and mature $\beta$ cells are not readily expanded in vitro. In this issue of the $J C I, O u$ et al. report that mature $\beta$ cell expansion can be induced in situ through epigenetic editing of regulatory elements in pancreatic tissue. Specifically, hypomethylation at imprinting control region 2 (ICR2) in human islets promoted $\beta$ cell expansion. Importantly, transplantation of these epigenetically edited islets into diabetic mice reduced blood glucose levels. Together, these results support further evaluation of this strategy for restoring $\beta$ cell mass in patients with diabetes.
\end{abstract}

\section{The challenge of restoring $\beta$ cell} function

Cell replication and identity are strictly controlled through multiple layers of mechanisms within tissues to maintain tissue homeostasis. In some cases, expansion of functional mature cells is needed to replace damaged cells in tissues. For type I or severe forms of type II diabetes, replenishment of malfunctioning $\beta$ cells with functional $\beta$ cells via islet transfer surgery has potential to confer long-term control of blood glucose levels. Unfortunately, there are few sources of healthy islet donors; therefore, additional strategies to restore $\beta$ cell function are needed.

\section{Engineered $\beta$ cells as an alternative to islet transplantation}

Beckwith-Wiedemann syndrome is an overgrowth disorder that features an excessive $\beta$ cell mass, and the DNA hypomethylation status of imprinting control region 2 (ICR2) has been linked to the massive $\beta$ cell expansion in these patients (1). This observation implies that manipulation of ICR2 has potential to modulate the expansion of mature $\beta$ cells. In this issue, Ou et al. test- ed this hypothesis and used a TALE-TET1 fusion protein to target and demethylate ICR2 (2). TALE-TET1-induced hypomethylation of ICR2 consequently resulted in reentry of mature $\beta$ cells into the cell cycle, thereby expanding the population. Moreover, transplantation of TALE-TET1expressing islets into immunodeficient diabetic mice restored insulin secretion to a level capable of reducing blood glucose.

The reactivation method used by Ou et al. is quite unique compared with cell transplantation (2), in which exogenous functional cells are transplanted into the liver or abdomen. Once injected, the transplanted cells are faced with harsh microenvironmental challenges that lead to poor survival of the donor cells and little restoration of $\beta$ cell function. Moreover, transplanted cells are at risk of being converted into a pathological phenotype, perhaps via interactions with locally secreted factors. Thus, reactivation of mature $\beta$ cells in situ can avoid the myriad problems mentioned above.

\section{Future directions and conclusions}

Promoter hypomethylation usually results in high levels of expression of the regu-

Related Article: p. 209

Conflict of interest: The authors have declared that no conflict of interest exists

Reference information: / Clin Invest. 2019;129(1):51-52. https://doi.org/10.1172/JCI124583.

lated genes (3-5); however, TALE-TET1mediated demethylation of ICR2 resulted in substantial suppression of p57. Although $\mathrm{Ou}$ and colleagues did not explore the underlying mechanisms of p57 suppression, several potential mechanisms could be further evaluated (2). For example, the issue can be examined from the standpoint of interactions between the ICR 2 and other regulatory regions of $\mathrm{p} 57$ in a 3-dimensional context. Hypomethylation may disrupt ICR2-involved DNA-DNA/DNA-IncRNA interactions that influence p57 expression. Also, it is possible that ICR2 hypomethylation impairs/enables binding of critical factors and that this impairment/enhancement leads to repression of p57. More work is needed to uncover how ICR2 methylation regulates p57 expression, as understanding the underlying mechanisms may be useful for precisely manipulating p57 expression and $\beta$ cell proliferation.

In addition to p57, other cell cycle inhibitors, such as p16, may be targets for increasing $\beta$ cell proliferation (6). Therefore, it would be interesting to determine if epigenetic repression of p16 expression also promotes expansion of mature $\beta$ cell populations in vivo. Alternatively, epigenetic editing methods could be applied to activate transcription factors to reprogram $\alpha$ cells into $\beta$ cells in vivo. Indeed, as proof of principle, recent studies have demonstrated the conversion of $\alpha$ cells into $\beta$ cells in vivo (7, 8). Further research is needed to determine whether or not CRISPR fusion proteins can be used to reprogram $\alpha$ cells in vivo.

In a broader sense, epigenetic modification of regulatory elements may provide a versatile platform to engineer cell fate in a variety of tissues in the field of regenerative medicine. For example, epigenetic targeting of DNA elements is critical for somatic cell reprogramming $(9,10)$, and the study by $\mathrm{Ou}$ et al. identifies a promising way to change cell fates with the use of synthetic tools (2). From this viewpoint, studies across different fields have already accumulated large data sets of epigenetic modifications, including histone, DNA, 
and RNA modifications, that are part of the epigenetic fingerprint associated with different cell statuses. Based on these data sets, it is reasonable to hypothesize that cell identities and/or physiological phenotypes are associated with these epigenetic characteristics. Thus, it is possible to engineer cell fate changes with fusion proteins between TALE or CRISPR and epigenetic enzymes or other proteins to modify these regions, and thereby modulate the expression of cell fate-associated genes. Similarly, as cancer cells harbor aberrant DNA methylation patterns or other epigenetic modifications $(11,12)$, a CRISPR-TET or CRISPR-X fusion protein could be designed to target single or multiple regions to modify epigenetic status and reprogram cancer cell fate. Compared with methods that introduce genes directly into cells, epigenetic editing can avoid risks of random insertion of exogenous genes into the genome; moreover, epigenetic editing provides much more flexible and precise control of target gene expression.

With the advancement of nanotechnology and/or other related delivery strat- egies, epigenetic targeting has potential to be expanded for many future applications in the field of regenerative medicine.

\section{Acknowledgments}

This work is supported by the Science and Technology Planning Project of Guangdong Province, China (2017B030314056).

Address correspondence to: Duanqing Pei, Guangzhou Institutes of Biomedicine and Health, CAS, No. 190, KaiYuan Road, Science Park, Guangzhou, Guangdong 510530, China. Phone: 86.20.32015231; Email:pei_duanqing@gibh.ac.cn.

1. Kalish JM, et al. Congenital hyperinsulinism in children with paternal 11p uniparental isodisomy and Beckwith-Wiedemann syndrome. J Med Genet. 2016;53(1):53-61.

2. Ou K, et al. Targeted demethylation at the CDKN1C/p57 locus induces human $\beta$ cell replication. JClin Invest. 2019;129(1):209-214.

3. Baylin SB, Jones PA. A decade of exploring the cancer epigenome - biological and translational implications. Nat Rev Cancer. 2011;11(10):726-734.

4. Bird A. DNA methylation patterns and epigene- tic memory. Genes Dev. 2002;16(1):6-21.

5. Maeder ML, et al. Targeted DNA demethylation and activation of endogenous genes using programmable TALE-TET1 fusion proteins. Nat Biotechnol. 2013;31(12):1137-1142.

6. Krishnamurthy J, et al. p16INK4a induces an age-dependent decline in islet regenerative potential. Nature. 2006;443(7110):453-457.

7. Thorel F, et al. Conversion of adult pancreatic $\alpha$-cells to $\beta$-cells after extreme $\beta$-cell loss. Nature. 2010;464(7292):1149-1154.

8. Xiao X, et al. Endogenous reprogramming of $\alpha$ cells into $\beta$ cells, induced by viral gene therapy, reverses autoimmune diabetes. Cell Stem Cell. 2018;22(1):78-90.e4.

9. Liu P, Chen M, Liu Y, Qi LS, Ding S. CRISPRbased chromatin remodeling of the endogenous Oct 4 or Sox 2 locus enables reprogramming to pluripotency. Cell Stem Cell. 2018;22(2):252-261.e4.

10. Gao X, Yang J, Tsang JC, Ooi J, Wu D, Liu P. Reprogramming to pluripotency using designer TALE transcription factors targeting enhancers. Stem Cell Reports. 2013;1(2):183-197.

11. Tse JWT, Jenkins LJ, Chionh F, Mariadason JM. Aberrant DNA methylation in colorectal cancer: what should we target? Trends Cancer. 2017;3(10):698-712

12. Klutstein M, Nejman D, Greenfield R, Cedar H. DNA methylation in cancer and aging. Cancer Res. 2016;76(12):3446-3450. 